\title{
Influences of Soret effect and Thermal Radiation on MHD Mixed Convective Mass Transfer Flow with Chemical Reaction and Heat Generation/Absorption
}

\author{
Sanjib Sengupta \\ Department of Mathematics, \\ Assam University, Silchar, \\ Assam, India
}

\begin{abstract}
The present paper deals with two dimensional steady laminar flow of a Newtonian electrically conducting incompressible viscous fluid past a permeable plate immersed in Darcian porous media in presence of thermal diffusion (Soret), thermal radiation, first order chemical reaction and heat generation / absorption effects. The exact solutions for velocity, temperature and concentration fields as well as skinfriction, Nusselt number and Sherwood number are obtained and discussed through graphs and tables in presence of various pertinent parameters involve in the study. The main velocity is found to increase due to increase in parametric values of thermal diffusion, permeability parameter, while the increase in Schmidt number and chemical reaction parameter decrease the flow rate. It is also seen that, the mass transfer rate accelerates under the influence of Schmidt number and chemical reaction parameter, whereas the skin-friction increases as thermal diffusion parameter increases and decreases due to increase in chemical reaction parameter.
\end{abstract}

\section{Keywords}

Mixed convection, thermal radiation, chemical reaction, Soret effect, heat source

\footnotetext{
Nomenclature

$\bar{C}$ Species concentration

$c_{p}$ Specific heat at constant pressure

$\bar{C}_{w}$ Species concentration at the plate

$\bar{C}_{\infty}$ Species concentration in the free stream

$D_{M}$ Co-efficient of mass diffusion

$D_{T}$ Co-efficient of thermal diffusion

$F \quad$ Non-dimensional chemical reaction parameter

$g \quad$ Acceleration due to gravity constant

Gm Thermal Grashof number

Gr Solutal Grashof number

$k \quad$ Thermal conductivity

$\bar{K}_{l}$ Non-dimensional chemical reaction parameter.

$\mathrm{Pr} \quad$ Prandtl number

$\bar{Q}_{l}$ Heat generation / absorption parameter (Dimensional)

$S$ Non-dimensional Heat generation / absorprion parameter

Sc Schmidt number

$\mathrm{Sr}$ Soret number

$t$ Time variable(Non-Dimensional)
}

$\bar{t}$ Time variable (Dimensional)

$\bar{T} \quad$ Fluid temperature

$\bar{T}_{w}$ Temperature at the plate

$\bar{T}_{\infty}$ Temperature in the free stream

$u$ First component of fluid velocity (Non-Dimensional)

$\bar{u}$ First component of fluid velocity(Dimensional)

$U_{0}$ Free stream velocity

y $\quad \mathrm{y}$-co-ordinate (Dimensional)

$\bar{y} \quad$ y coordinate (Non-Dimensional)

$v$ Second component of fluid velocity (Non-Dimensional)

$V_{0}$ Mean suction velocity

$\bar{v}$ Second component of fluid velocity (Dimensional)

\section{Greek Symbols}

$\beta$ Thermal Coefficient of volumetric expansion

$\beta^{*}$ Solutal Coefficient of volumetric expansion

$\rho$ Fluid density

$v \quad$ Kinematic coefficient of viscosity

$\theta \quad$ Non-dimensional temperatur

$\phi \quad$ Non-dimensional species concentration

\section{Subscripts \\ $w \quad$ Conditions on the walls \\ $\infty \quad$ Free stream conditions}

\section{INTRODUCTION}

The theory of heat and mass transfer flow with mixed convection is constantly developing due to its importance in space technology, meteorological sciences and particularly in nuclear reactors. The process of convection heat transfer associated with a heated/cooled vertical plate is one of the fundamental problems in heat and mass transfer studies and thus extensive research has been done in this field by the researchers. If, a free convective flow is accompanied by an external flow, itis commonly called as mixed convection. Chamkha et al. (2004)studied the effects of localized heating (cooling), suction (injection), buoyancy forces and magnetic 
field for the mixed convection flow on a heated vertical plate. Abdelkhalek(2006)considered the MHD mixed convective stagnation point flow impinging on a heated vertical semiinfinite permeable surface. Aydin and Kaya (2007) studied the mixed convection of viscous dissipating fluid about a vertical plate. Sengupta (2012)investigated the case of radiative mixed convective mass transfer flow in presence of thermal diffusion and heat generation.

At very high temperatures many technological processes take place, where the thermal radiation heat transfer becomes very important and its effects cannot be ignored. Recent developments in hypersonic flights, missile reentry rocket combustion chambers, gas cooled nuclear reactors and power plants for inter planetary flight have focused attention of researchers on thermal radiation as a mode of energy transfer and emphasize the need for inclusion of radiative transfer in these process. The knowledge of radiative heat transfer also becomes very significant especially in electronics equipment and many processes in industry, which occur at very high temperatures. Cess (1966) investigated radiation effects on free convective heat transfer flow. The radiation effect on a natural convective flow of an absorbing emitting liquid was considered by Novotny et al. (1977).Sattar and Kalim (1996) had investigated unsteady free convection interaction with thermal radiation in a boundary layer flow past a vertical porous plate. The case of unsteady flow in presence of radiation and variable viscosity on a MHD flow past a semiinfinite flat plate with an aligned magnetic field was studied by Seddeek (2002). Very recently, Sengupta (2014) obtained closed form of solution of the influence of thermal radiation effect on an unsteady free convective chemically absorption fluid through past an impulsively accelerated plate in presence of a transverse magnetic field.

In contrary, the theory of heat and mass transfer with chemical reaction is of considerable importance in the chemical and hydrometallurgical industries. Chambre and Young (1958) have analyzed a first order chemical reaction in the neighborhood of a horizontal plate. Muthucumaraswamy et al. (2002) considered the effects of chemical reaction on a moving isothermal surface with suction. Akyildizet al. (2006) studied the diffusion of chemically reactive species in a porous medium over a stretching sheet. Sengupta and Sen (2013) investigated first order chemical reaction effect on a free convective absorbing fluid in presence of heat sink and uniform heat flux.

It is observed that, a mass flux may be generated by the effects of both concentration gradients as well as by the temperature gradients. Mass flux influenced by temperature gradient is termed as Soret or thermal diffusion effect. The pioneering work in Soret effect was made by Eckert and Drake (1972).Notable contributions in thermo - diffusion effects are also made by researchers like, Afify (2009) and Hayat et al. (2010) etc. Sengupta (2011) considered the influence of thermal diffusion effect on a free convective mass transfer flow with heat sink.

The objective of the present paper is to investigate the effects of first order chemical reaction on a steady two-dimensional mixed convective flow of a laminar incompressible viscous Newtonian fluid in presence of thermal diffusion and heat generation / absorption.

\section{MATHEMATICAL FORMULATION AND SOLUTION OF THE PROBLEM}

A co-ordinate system $(\overline{\boldsymbol{x}}, \overline{\boldsymbol{y}})$ has been introduced, with its $\bar{x}$-axis along thelength of the infinite plate in the upward vertical direction and $\bar{y}$-axis perpendicular to the plate towards the fluid region. The flow field is considered to be steady, laminar and two-dimensional, whereas the fluid and the porous media are in local thermo-dynamical equilibrium. A uniform magnetic field of moderate strength is applied transversely to the plate surface, which is subjected to a constant suction velocity. Using Boussinesq approximation, a two- dimensional fully developed fluid model has been established in terms of a system of coupled partial differential equations, combined with two-point semi-open boundary conditions as:

\section{Continuity Equation}

$\frac{\partial \bar{v}}{\partial \bar{y}}=0$

\section{Momentum Equation}

$\bar{v} \frac{\partial \bar{u}}{\partial \bar{y}}=-\frac{1}{\rho} \frac{\partial \bar{p}}{\partial \bar{x}}+v \frac{\partial^{2} \bar{u}}{\partial \bar{y}^{2}}+g \beta\left(\bar{T}-\bar{T}_{\infty}\right)+g \beta^{*}\left(\bar{C}-\bar{C}_{\infty}\right)-\left(\frac{\sigma B_{0}{ }^{2}}{\rho}+\frac{v}{\bar{K}}\right) \bar{u}$

\section{Energy Equation}

$\bar{v} \frac{\partial \bar{T}}{\partial \bar{y}}=\frac{k}{\rho c_{p}} \frac{\partial^{2} \bar{T}}{\partial \bar{y}^{2}}-\frac{1}{\rho c_{p}} \frac{\partial \bar{q}_{r \bar{y}}}{\partial \bar{y}}+\frac{\bar{Q}_{l}}{\rho c_{p}}\left(\bar{T}-\bar{T}_{\infty}\right)$

\section{Species Continuity Equation}

$\bar{v} \frac{\partial \bar{C}}{\partial y}=D_{M} \frac{\partial^{2} \bar{C}}{\partial \bar{y}^{2}}+D_{T} \frac{\partial^{2} \bar{T}}{\partial \bar{y}^{2}}-K_{l}\left(\bar{C}-\bar{C}_{\infty}\right)$

Subject to the relevant boundary conditions as:

$$
\begin{aligned}
& \bar{u}=0, \bar{v}=V_{0}, \bar{T}=\bar{T}_{w}, \bar{C}=\bar{C}_{w}, \text { at } \bar{y}=0 \\
& \bar{u} \rightarrow \bar{U}, \bar{v}=0 \bar{T} \rightarrow \bar{T}_{\infty}, \bar{C} \rightarrow \bar{C}_{\infty} \text {, at } \bar{y}=0
\end{aligned}
$$

Using Bernoulli's pressure equation, the total (mechanical) pressure of the flow field can be quantified as:

$$
-\frac{1}{\rho} \frac{\partial \bar{p}}{\partial \bar{x}}=\frac{\nu \bar{U}}{\bar{K}}
$$

By virtue of (6), equation (2) can recast as:

$\bar{v} \frac{\partial \bar{u}}{\partial \bar{y}}=v \frac{\partial^{2} \bar{u}}{\partial \bar{y}^{2}}+g \beta\left(\bar{T}-\bar{T}_{\infty}\right)+g \beta^{*}\left(\bar{C}-\bar{C}_{\infty}\right)+\left(\frac{\sigma B_{0}{ }^{2}}{\rho}+\frac{v}{\bar{K}}\right)\left(U_{0}-u\right)$

The constant suction velocity can be considered as:

$\bar{v}(t)=-V_{0},\left(V_{0}>0\right)$

Following Magyari and Pentokratoras (2011), the Rosseland approximation model quantifying the radiative heat flux for an optically thick boundary layer flow, in a simplified differential form is considered as:

$\bar{q}_{r}=-\frac{4 \sigma^{*}}{3 k^{*}} \frac{\partial \bar{T}^{4}}{\partial \bar{y}}$

If, the temperature differences within the flow are sufficiently small, so that, $\bar{T}^{4}$ may be expressed as a linear function of temperature $\bar{T}$ and expanding $\bar{T}^{4}$ in Taylor's series about $\bar{T}_{\infty}$ and neglecting higher order terms we thus derive,

$\bar{T}^{4} \approx \bar{T}_{\infty}^{4}+\left(\bar{T}-\bar{T}_{\infty}\right) 4 \bar{T}_{\infty}^{3}=4 \bar{T}_{\infty}^{3} \bar{T}-3 \bar{T}_{\infty}^{4}$ 
Using (9) and (10), equation (3) becomes,

$\bar{v} \frac{\partial \bar{T}}{\partial \bar{y}}=\frac{k}{\rho c_{p}} \frac{\partial^{2} \bar{T}}{\partial \bar{y}^{2}}-\frac{16 \sigma^{*} \bar{T}_{\infty}^{3}}{3 \rho c_{p} k^{*}} \frac{\partial^{2} \bar{T}}{\partial \bar{y}^{2}}+\frac{\bar{Q}_{l}}{\rho c_{p}}\left(\bar{T}-\bar{T}_{\infty}\right)$

Introduce the following non-dimensional quantities as:

$y=\frac{\bar{y} V_{0}}{v}, U_{0}=\frac{\bar{U}}{V_{0}}, u=\frac{\bar{u}}{U_{0}}, v=\frac{\bar{v}}{V_{0}}$,

$\theta=\frac{\bar{T}-\bar{T}_{\infty}}{\bar{T}_{w}-\bar{T}_{\infty}}, \phi=\frac{\bar{C}-\bar{C}_{\infty}}{\bar{C}_{w}-\bar{C}_{\infty}}, G r=\frac{g \beta v\left(\bar{T}_{w}-\bar{T}_{\infty}\right)}{V_{0}^{2} U_{0}}$,

$M=\frac{\sigma B_{0}{ }^{2} v}{\rho V_{0}{ }^{2}} S c=\frac{v}{D_{M}} G m=\frac{g \beta v\left(\bar{C}_{w}-\bar{C}_{\infty}\right)}{V_{0}{ }^{2} U_{0}}$,

$K=\frac{V_{0}^{2} \bar{K}}{v^{2}}, \operatorname{Pr}=\frac{v \rho c_{p}}{k}, S r=\frac{D_{T}\left(\bar{T}_{w}-\bar{T}_{\infty}\right)}{v\left(\bar{C}_{w}-\bar{C}_{\infty}\right)}$,

$Q_{0}=\frac{\bar{Q}_{l} v^{2}}{k V_{0}^{2}} \quad F=\frac{\bar{K}_{l} v}{V_{0}^{2}}$.

The non-dimensional form of equations (7), (3) and (4) is,

$\frac{d^{2} u}{d y^{2}}+\frac{d u}{d y}-M_{1} u=-M_{1} U_{0}-G r \theta-G m \phi$

$\lambda \frac{d^{2} \theta}{d y^{2}}+\operatorname{Pr} \frac{d \theta}{d y}+Q_{0} \theta=0$

$\frac{d^{2} \phi}{d y^{2}}+S c \frac{d \phi}{d y}-F S c \phi=-S r S c \theta$

Where, $M_{1}=M+\frac{1}{K}$ and $\lambda=1+\frac{4 R}{3}$ (say).

The corresponding non-dimensional initio - boundary conditions are:

$u(0)=0, \theta(0)=1, \phi(0)=1$,

$u(\infty) \rightarrow U_{0}, \theta(\infty) \rightarrow 0, \phi(\infty) \rightarrow 0$

The exact analytical solution of equations (12)-(14) subject to (15.1) - (15.2) is thus calculated as:

$\theta(y)=\exp \left(-\gamma_{1} y\right)$

$\phi(y)=\eta_{1} \exp \left(-\gamma_{1} y\right)+\eta_{2} \exp \left(-\gamma_{2} y\right)$

$u(y)=U_{0}+\eta_{3} \exp \left(-\gamma_{1} y\right)+\eta_{4} \exp \left(-\gamma_{2} y\right)$

Skin-friction at the plate:

$\tau=\left(\frac{\partial u}{\partial y}\right)_{y=0}=\gamma_{1} \eta_{3}+\gamma_{2} \eta_{4}$

Nusselt number at the plate:

$N u=-\frac{1}{\operatorname{Pr}}\left(\frac{\partial \theta}{\partial y}\right)_{y=0}=\frac{\gamma_{1}}{\operatorname{Pr}}$

Sherwood number at the plate:

$S h=-\frac{1}{S c}\left(\frac{\partial \phi}{\partial y}\right)_{y=0}=\frac{1}{S c}\left(\gamma_{1} \eta_{1}+\gamma_{2} \eta_{2}\right)$

Where, $\gamma_{1}=\frac{\operatorname{Pr}+\sqrt{\operatorname{Pr}^{2}-4 \lambda Q_{0}}}{2 \lambda},\left(\operatorname{Pr}^{2}>4 \lambda Q_{0}\right)$,

$\gamma_{2}=\frac{S c+\sqrt{S c^{2}+4 F S c}}{2}, \eta_{1}=-\frac{S r S c}{\gamma_{1}^{2}-S c \gamma_{1}-F S c}$, $\eta_{2}=1-\eta_{1}, \quad \eta_{3}=-\frac{G r+\eta_{1} G m}{\gamma_{1}^{2}-\gamma_{1}-M_{1}}, \quad \eta_{4}=-\frac{G m\left(1+\eta_{1}\right)}{\gamma_{2}^{2}-\gamma_{2}-M_{1}}$.

\section{RESULTS AND DISCUSSION}

The parametric influence of various physical parameters on the velocity, temperature and concentration profiles as well as on the Nusselt and Sherwood numbers are presented through graphs 1-9.

Figure 1 depicts the influence of chemical reaction parameter $(F)$ on the concentration profiles $(\phi, y)$ for a set of fixed values of $\operatorname{Pr}=7.0, Q_{0}=0.5, S r=0.5, R=0.375, y=0.02$. Due to increase in $F$, the constituents from higher concentration zone (adherent to the plate)moves towards the species in lower concentration zone (free stream) results of which decreases the concentration boundary layer thickness,thus decreasing the values of $\phi$. Figure 2 presents the schematic presentation of the influence of thermal radiation $(R)$ on the concentration profiles $(\phi, y)$ for a set of fixed values of $\operatorname{Pr}=7.0, Q_{0}=0.5$, $S r=0.5, F=0.5, y=0.02$. The increase in values of $R$ is found to increase the concentration near the plate.

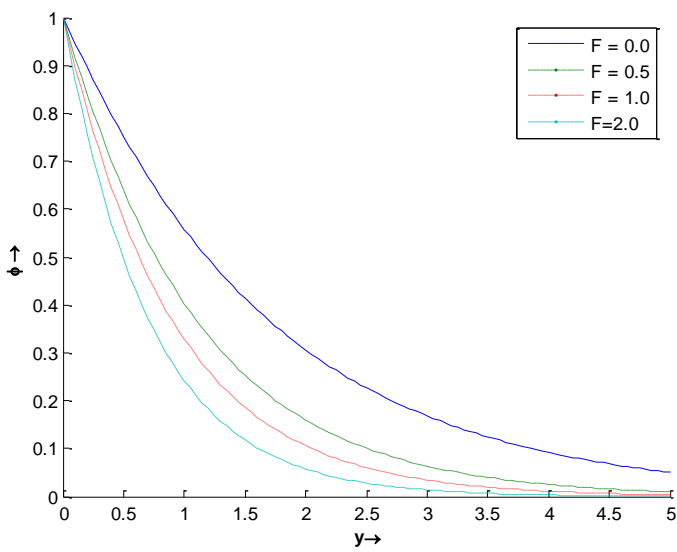

Figure1 $\phi$ versus $y$ for change in values of $\boldsymbol{F}$

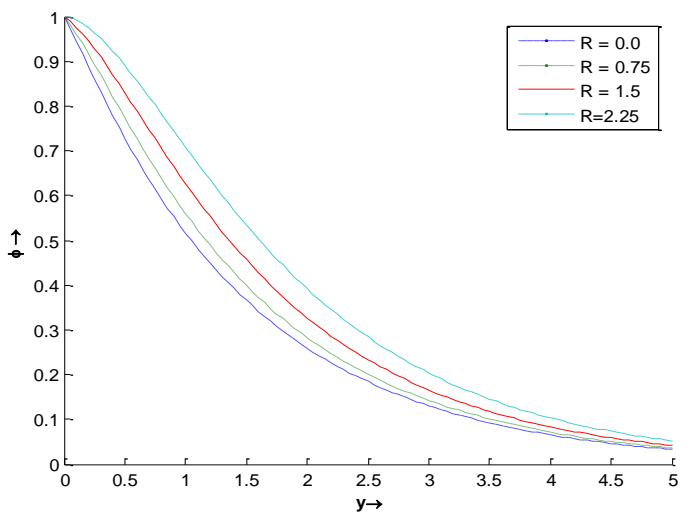

Figure2 $\phi$ versus $\boldsymbol{y}$ for change in values of $\boldsymbol{R}$.

Figures 3 to 6 show how the flow rate are being influenced by the presence of parameters like, Soret number $(S r)$, magnetic field parameter $(M)$, permeability parameter $(K)$ and chemical reaction parameter $(F)$ against normal distance $y$ for a set of 
fixed values of $\operatorname{Pr}=7.0, Q_{0}=0.5, U_{0}=0.5, S c=0.60, F=0.5$, $G r=5.0, G m=2.0, R=0.375$ (except for fig. 3) as well as $S r=$ 0.5 ( for figs. 4,5, 6), $M=0.5$ ( for figs. 3, 5,6), $K=0.5$ ( for figs. $3,4,6$ ) and $F$ (for figs. $3,4,5$ ). It is observed that, the flow rate accelerates due to increase in values of $S r$ and $K$ respectively and it is found decelerating as the values of $M$ and $F$ increase. Due to increase in $S r$, the mass buoyancy forces increase which accelerates the flow rate and thus increase the value of $u$. Also as the values of $K$ increase, the frictional force decreases, this increases the flow rate and the fluid velocity $u$. Again, due to increase in $M$, the strength of the resistive force, known as Lorentz force (which generates along the flow due to the presence of the magnetic field), increases thus decelerates the flow rate and decreases the values of $u$. Due to increase in values of $F$, the concentration of fluid particles near the plate drops, which results in decreasing the effect of mass buoyancy forces and thus decrease the fluid velocity $u$.

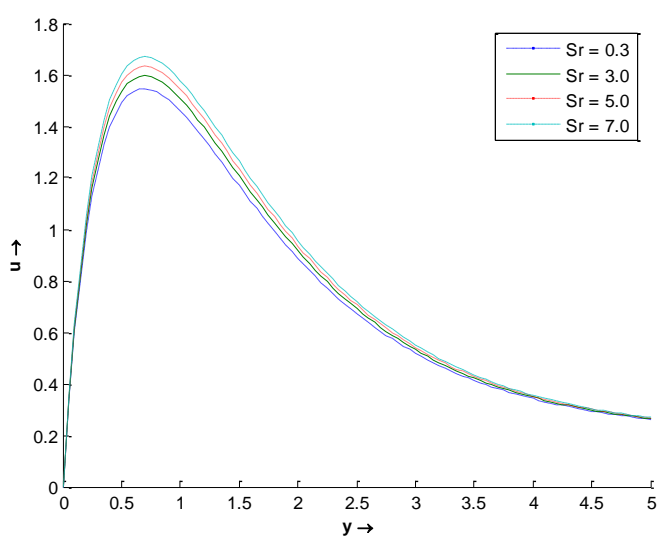

Figure3 u versus $y$ for change in values of $S r$

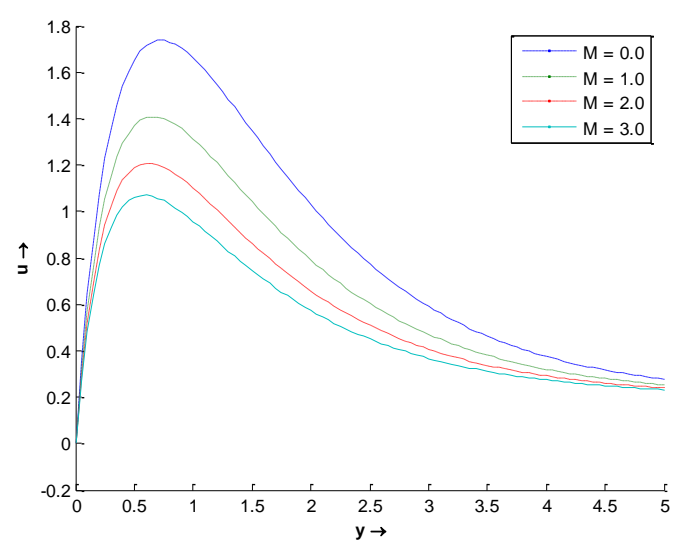

Figure4 $u$ versus $y$ for change in values of $M$

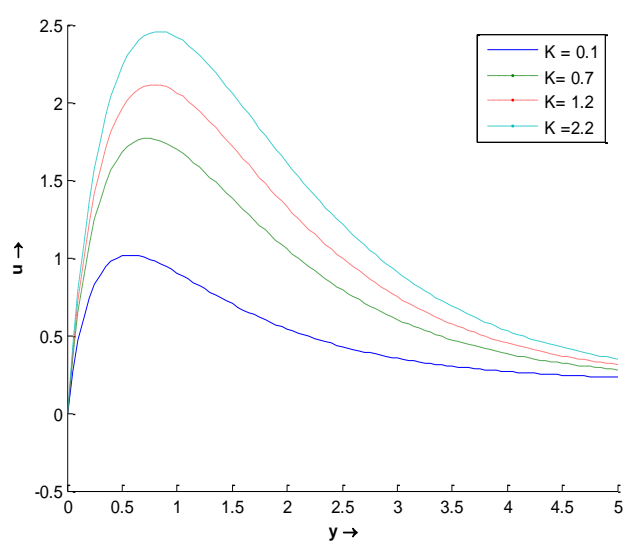

Figure 5 u versus $y$ for change in values of $K$

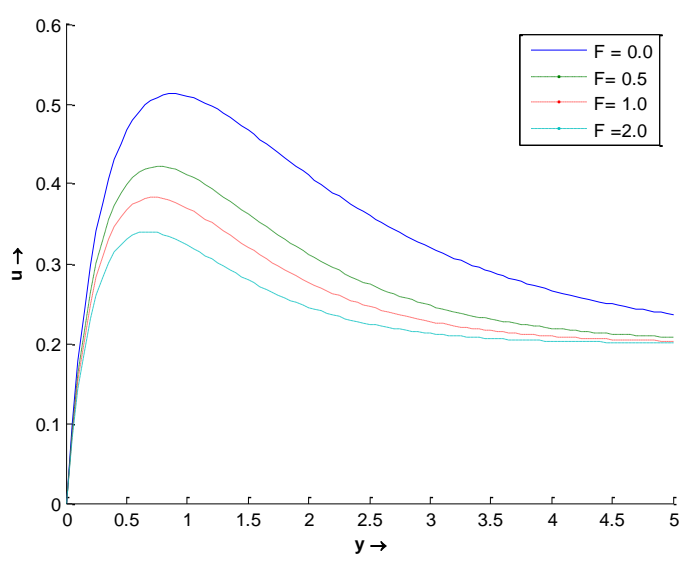

Figure 6 u versus $y$ for change in values of $F$

The influence of magnetic parameter $(M)$ on the skin-friction against chemical reaction parameter $(F)$ for a set of fixed values of $\operatorname{Pr}=7.0, \quad Q_{0}=0.2, U_{0}=0.2, S c=0.60, \quad G r=5.0$, $G m=2.0, R=0.375, S r=0.5, K=0$ is depicted in figure 7. It is observed that, the skin-frictional effect on the plate decreases due to increase in $M$ and $F$ respectively. The presence of both $M$ and $F$, decelerate the flow rate, which results in decreasing the frictional effect on the plate.

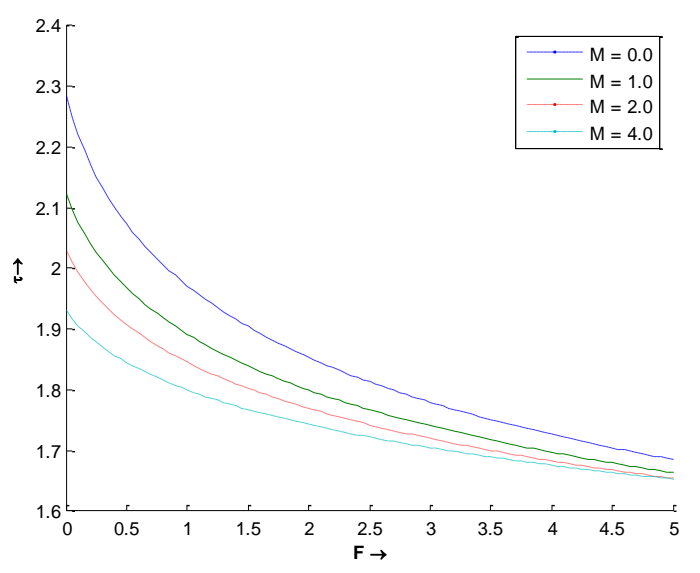

Figure $7 \tau$ versus $\boldsymbol{F}$ for change in values of $\boldsymbol{M}$ 
Figure 8 shows the influence of thermal radiation $(R)$ on the Nusselt number profiles $\left(N u, Q_{0}\right)$. When $Q_{0} \in[-1,0)$ i.e. in presence of heat sink parameter, the Nusselt number is found increasing due to increase in values of $R$, while for $Q_{0} \in[0,1]$ i.e. in presence of heat source parameter an opposite trend has observed for $\mathrm{Nu}$. Figure 9 presents how the Sherwood number is being influenced due to change in values of Soret number $(S r)$ against chemical reaction parameter $(F)$. It is seen that, the mass transfer rate gets accelerate as the values of $F$ increase. The cause of transfer of constituents from higher concentration zone to the lower concentration zone due to chemical reaction parameter increases the values of $S h$. Again, as the values of $S r$ increase, the value of $S h$ decreases which is in support of the fact that, the thickness of the concentration boundary layer raises duo to increase in $S r$, which drops the mass transfer rate within the flow.

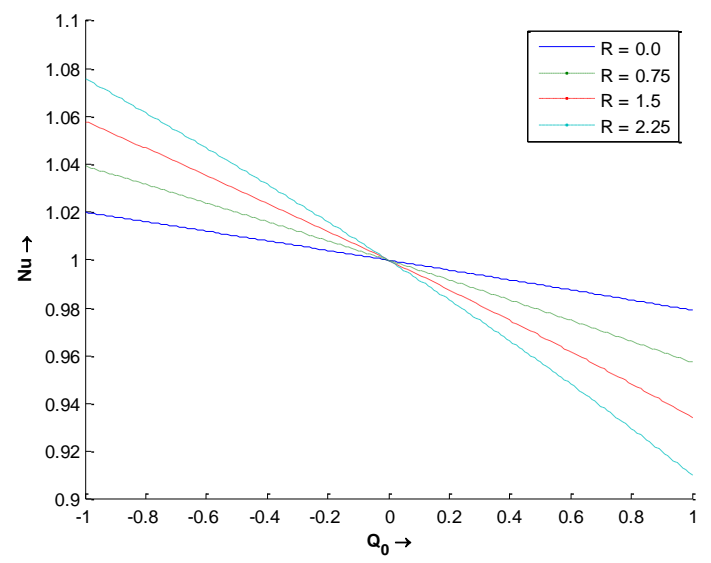

Figure $8 \mathrm{Nu}$ versus $Q_{0}$ for change in values of $\mathrm{R}$

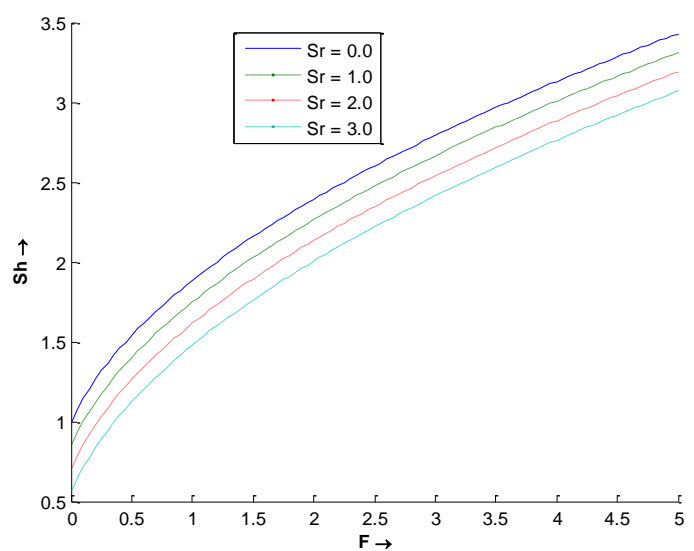

Figure9 $S h$ versus $F$ for change in values of $S r$

\section{CONCLUSIONS}

The significant outcome of the study is highlighted as follows:

- The concentration increases as thermal radiation parameter increases, while concentration drops due to increase in chemical reaction parameter.

- The velocity of flow increases as Soret number and permeability parameters increase, while the increase in magnetic parameter and chemical reaction parameter is found to decrease the fluid velocity.

- The Nusselt number first increases in presence of heat absorption parameter and thereafter it is found decreasing in presence of heat generation parameter due to increase in values of thermal radiation parameter.

- The Sherwood number increases as Soret number increases in presence of chemical reaction parameter.

- The skin-friction decreases as magnetic parameter increases in presence of chemical reaction parameter.

\section{ACKNOWLEDGMENT}

The author is thankful to the reviewers for providing constructive suggestions.

\section{REFERENCES}

[1] Abdelkhalek, M.M. The skin friction in the MHD mixed convection stagnation point with mass transfer, Int. Commun. Heat Mass 33(2006), 249-258.

[2] Afify, A.A. Similarity solution in MHD: Effects of thermal diffusion and diffusion- thermo on free convective heat and mass transfer over a stretching surface considering suction or injection, Communication in Non-linear Science and numerical simulation, 14(2009), 2202-2214.

[3] Akyildiz, F. T., Bellout H, Vajravelu K. Diffusion of chemically reactive species in a porous medium over a stretching sheet. J. Math Anal Appl320(2006), 322-39.

[4] Aydin, O., Kaya, AMixed convection of a viscous dissipating fluid about a vertical flat plate, Appl. Math. Model 31(2007), 843-853.

[5] Cess,R. D. The Interaction of Thermal Radiation with Free Convection HeatTransfer, Int. J. Heat Mass Trans., 9(1966), 269-277.

[6] Chambre PL. and Young JD On the diffusion of a chemically reactive species in a laminar boundary layer flow, the Physics of Fluids. 1(1958), 48-54.

[7] Chamkha, A.J. Takhar, H.S. Nat, GMixed convection flow over a vertical plate with localized heating (cooling), magnetic field and suction (injection),Heat Mass Transfer 40(2004), 835-841.

[8] Eckert, E. R. G. and Drake, R. M. Analysis of heat and mass transfer, Hemisphere Pub. Corp., Washington, D.C., 1972.

[9] Hayat T, Mustafa M and Pop I. Heat and mass transfer for Soret and Dufour's effect on mixed convection boundary layer flow over a stretching vertical surface in a porous medium filled with viscoelastic fluid. Commun Nonlinear SciNumer Simul, 15(2010), 1183-1196.

[10] Magyari E.and Pentokratoras, A. Note on the effect of thermal radiation in the linearized Rosseland approximation on the heat transfer characteristics of various boundary layer flow, Int. commun. heat and mass transfer, 38(2011), 554.

[11] Muthecumaraswamy R, Effects of a chemical reaction on a moving isothermal surface with suction. ActaMechanica 155(2002), 65-72.

[12] Novotny, J. L., Lloyd, J .R. and Bankston, J. D. 
Radiation Convection Interaction in an Absorbing Emitting Liquid in Natural Convection Boundary Layer Flow, ASME, J. Heat Transfer, 99(1977),125-127.

[13] Satter, A. M. D. and Kalim,H.M.D. Unsteady freeconvection interaction with thermal radiation in a boundary layer flow past a vertical porous plate, J Math PhysSci, 30(1996), 25-37.

[14] Seddeek, M.A.: Effects of radiation and variable viscosity on a MHD free convection past a semi-infinite flat plate with an aligned magnetic field in thecase of unsteady flow, Int J Heat Mass Transfer, 45(2002),931935.

[15] Sengupta, S. Thermal diffusion effect of free convection mass transfer flow past a uniformly accelerated porous plate with heat sink, International Journal of Mathematical Archive, 2(2011), 1266-1273.
[16] Sengupta, S. Radiative mixed convection mass transfer flow past an iso-thermal porous plate embedded in a permeable medium in presence of thermal diffusion and heat generation, International Journal of Computer Applications, 40(2012), 17-22.

[17] Sengupta, S. Unsteady MHD Free Convective Chemically Absorption Fluid Past an Impulsively Accelerated Plate with thermal radiation, Int. J. for research in appl. Science and Engg., 2 (XII) (2014), 91101.

[18] Sengupta, S. and Sen, M. Free convective heat and mass transfer flow past an oscillating plate with heat generation, thermal radiation and thermo-diffusion effects, JP J. of heat and mass transfer, 8(2)(2013), 187210. 